\title{
An exploration and comparison of food and drink availability in homes in a sample of families of White and Pakistani origin within the UK
}

\author{
Maria Bryant ${ }^{1,2, *}$, Pinki Sahota ${ }^{3}$, Gillian Santorelli $^{2}$ and Andrew Hill ${ }^{4}$ \\ ${ }^{1}$ Leeds Institute of Clinical Trials Research, University of Leeds, Leeds LS2 9JT, UK: ${ }^{2}$ Institute of Health Research, \\ Bradford Teaching Hospitals NHS Trust, Bradford, UK: ${ }^{3}$ Nutrition and Dietetics, Leeds Metropolitan University, \\ Leeds, UK: ${ }^{4}$ Leeds Institute of Health Sciences, University of Leeds, Leeds, UK
}

Submitted 9 September 2013: Final revision received 17 December 2013: Accepted 30 January 2014: First published online 10 March 2014

\begin{abstract}
Objective: Knowledge of the types and quantities of foods and drinks available in family homes supports the development of targeted intervention programmes for obesity prevention or management, or for overall diet improvement. In the UK, contemporary data on foods that are available within family homes are lacking. The present study aimed to explore home food and drink availability in UK homes.

Design: An exploratory study using researcher-conducted home food availability inventories, measuring all foods and drinks within the categories of fruits, vegetables, snack foods and beverages.

Setting: Bradford, a town in the north of the UK.

Subjects: Opportunistic sample of mixed ethnicity families with infants approximately 18 months old from the Born in Bradford birth cohort.

Results: All homes had at least one type of fruit, vegetable and snack available. Fresh fruits commonly available were oranges, bananas, apples, satsumas and grapes. Commonly available fresh vegetables included potatoes, cucumber, tomatoes and carrots. The single greatest non-fresh fruit available in homes was raisins. Non-fresh vegetables contributing the most were frozen mixed vegetables, tinned tomatoes and tinned peas. Ethnic differences were found for the availability of fresh fruits and sugar-sweetened beverages, which were both found in higher amounts in Pakistani homes compared with White homes.

Conclusions: These data contribute to international data on availability and provide an insight into food availability within family homes in the UK. They have also supported a needs assessment of the development of a culturally specific obesity prevention intervention in which fruits and vegetables and sugarsweetened beverages are targeted.
\end{abstract}

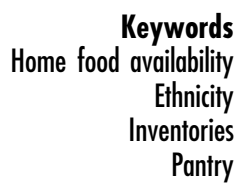

There has been increasing interest in the role that food availability in the home has on food consumption and obesity $^{(1-7)}$. Such information has the potential to increase understanding of the causes of energy overconsumption and provide direction to help create effective obesity prevention interventions. However, research in this area is limited by the methods used to assess food availability. Much of the literature uses data collected using food checklists; a quick and relatively inexpensive method to assess the presence or absence of a predefined list of selected foods using participant self-report. Data collected using this method are limited to the items that have been predefined and cannot therefore capture information on ethnically diverse habits or unexpected patterns. An alternative method to self-report checklists is to conduct researcher-administered inventories of the home food environment. These involve researchers going into participants' homes and recording all foods and drinks available (fully exhaustive inventories) or all foods and drinks available within predefined categories (partially exhaustive inventories). They are not limited to recording only foods that the researchers have previously assumed to be available in a predefined list. However, few attempts have been made to collect this kind of data owing to issues related to the feasibility of collection and analysis

A review of methods to collect home food availability data in $2006^{(8)}$ identified just three other published studies that had used fully or partially exhaustive inventories since 1975. Since then, some investigators have used 
this approach ${ }^{(9-11)}$; however, the majority of work has continued to focus on data collection using predefined checklists $^{(5,6,12)}$. This work has so far been dominated by the USA and indicates that the availability of foods is generally related to consumption in infants and children ${ }^{(5,13-15)}$ and weight status ${ }^{(2,5,6)}$, although findings are somewhat equivocal, likely due to the methodologies employed.

To our knowledge, there are no published studies describing home food availability collected using a researcher-conducted approach within populations in the UK. We aimed to explore the home food environment as part of an objective to develop a culturally appropriate obesity prevention intervention within the Born in Bradford-1000 Programme of research ${ }^{(16)}$. Since no other data of this kind have been collected in a mixed ethnic sample in the UK (and thus no appropriate checklist was available), open inventories of foods and drinks within pre-specified categories of fruits, vegetables, snack foods and drinks were conducted by researchers within participants' homes using a well-defined protocol already tested by the authors as part of their work in the $\mathrm{USA}^{(9,10)}$. The present study reports our findings from the inventories to explore which foods were available in the homes of a sample of families of mixed ethnicities when their infants were approximately 18 months old and to identify any differences in availability between White British and Pakistani homes.

\section{Experimental methods}

\section{Sample}

Participants were opportunistically recruited from Born in Bradford-1000 (BiB1000), a nested cohort within Born in Bradford (BiB). BiB is a longitudinal multi-ethnic birth cohort aiming to examine environmental, psychological and genetic factors that impact on health and development perinatally, during childhood and subsequent adult life, and those that influence their parents' health and well-being. All mothers booked in for a delivery of their baby in Bradford Royal Infirmary from March 2007 to December 2010 were invited to take part in the research during their routine glucose tolerance test at 26-28 weeks. A total of 12453 pregnant women enrolled who subsequently gave birth to 13776 babies. A full account of the methods is published elsewhere ${ }^{(17)}$. All mothers recruited to the main $\mathrm{BiB}$ cohort study between August 2008 and March 2009 (and who had completed the baseline questionnaire) were approached to take part in BiB1000 and a total of 1736 agreed. One hundred participants were then drawn opportunistically from the BiB1000 cohort to take part in the current food availability study during the 18-month BiB1000 assessment in which all approached agreed to take part. Due to the exploratory nature of the research, a formal sample size calculation was not performed. Inventory data from 100 homes were chosen as this was considered comparable to previous research ${ }^{(8)}$; within calculations to detect small to moderate group differences ${ }^{(18)}$; and due to issues of feasibility.

The study was conducted according to the guidelines laid down in the Declaration of Helsinki and all procedures involving human subjects/patients were approved by the Bradford Research Ethics Committee (07/H1302/ 112). Written or verbal (for mothers unable to read and/or speak English) informed consent was obtained from all participants. Verbal consent was witnessed and formally recorded.

\section{Data collection}

\section{Researcher-conducted food availability inventories}

Researcher-conducted inventories were conducted in 100 homes when infants were approximately 18 months old. Data collection method, staff training and quality assurance were conducted using a standardised protocol using well-established methodologies from previous research $^{(9,10)}$. Participants were told that researchers would be visiting their homes and that they would need access to all places in their homes were foods were stored. No incentives were provided. Researchers measured the availability (yes/no), quantity and size of all foods from all food storage areas in participants' homes within the higher categories of fruit (with sub-categories: fresh, tinned, dried and frozen), vegetables (with subcategories: fresh, tinned and frozen), snack foods (with sub-categories: crisps/tortillas, biscuits, salted nuts, chocolate, sweets, cakes and ice cream) and beverages (with sub-categories: sugar-sweetened and sugar-free). These categories were chosen because: (i) they are often the target of obesity interventions; (ii) there is some evidence that their intake is related to obesity in children ${ }^{(19,20)}$; and/or (iii) literature indicates a relationship between availability in the home and either diet ${ }^{(3,21)}$ or obesity ${ }^{(6,22)}$. Our previous studies also indicated that these items could be reliably and validly collected ${ }^{(9,10)}$. Within each subcategory, open 'exhaustive' data were collected rather than using a predefined checklist of items (i.e. details on all of the available foods and drinks available were recorded). This method was used as this was an exploratory study with no a priori data to suggest the nature or type of foods which were present in the homes of this sample. For fresh produce, researchers recorded the number of whole pieces (e.g. apples) or the number of handfuls (e.g. grapes). For non-fresh items, researchers recorded the number of foods and drinks within prespecified size ranges of small, medium and large units. These were defined by weight and were based on data previously collected ${ }^{(9)}$ plus the actual package sizes available to purchase in the UK. For example, tinned vegetables that weighed less than $250 \mathrm{~g}$ were defined as small; those weighing between 250 and $450 \mathrm{~g}$ were considered to be medium; and any weighing more than $450 \mathrm{~g}$ were defined as large. 


\section{Other measures pertinent to these analyses}

The majority of demographic data were obtained at recruitment (26-28 weeks of pregnancy) including: household structure; marital status; residence type; educational status; and ethnicity. Maternal smoking behaviour was ascertained at this point to determine whether participants were currently smoking during pregnancy by self-report. All questionnaires were transliterated into Urdu and Mirpuri language, as the majority of Pakistani populations residing in Bradford are of Mirpuri origin and one of the official languages of Pakistan is Urdu. The process of transliteration involved translation, back-translation and several rounds of piloting by bilingual and monolingual groups in collaboration with local experts in Bradford (Bradford Talking Media). Since Mirpuri does not have a written form, transliterations were made available for administration by bilingual study administrators. There were no language restrictions for eligibility into the present study and bilingual staff were trained to collect data from homes in which the parents were unable to speak English.

\section{Data cleaning}

Open, exhaustive data from 836 food and drink items that were identified within the homes of this sample were grouped to 215 individual food and drink types by a nutritionist (M.B.). For example, a packet of chocolate digestive biscuits' was grouped as 'biscuits with chocolate topping' within the sub-category of 'biscuits/sweet snacks' (under the higher category of snacks). Similarly, all crisps that were made with corn were assigned to the group of 'tortillas' within 'crisps/savoury snacks' and 'red grapes' and 'green grapes' were grouped as 'grapes' within the sub-category of fresh fruits (under the higher category of fruit). For the purpose of these analyses, one handful of fresh produce represented one serving. Other fresh produce that were recorded as whole units (e.g. melons) were converted to the number of servings by a nutritionist (M.B.) using standards provided by NutritionData.com (http://nutritiondata.self.com/facts) and the US Department of Agriculture (http://ndb.nal.usda.gov/ $\mathrm{ndb} /$ foods). Scores were generated for the analysis of non-fresh produce based on the number of each food item within predefined sizes. Small items were assigned a score of 1 per item; medium, a score of 2; and large, a score of 3 per item. These can be viewed as equivalent to the total number of small sized items. For example, a score of 4 for tinned vegetables is equivalent to having four small tins of vegetables in the home, even though it may have actually have been available as one large tin (score of 3) plus one small tin (score of 1).

\section{Statistical analysis}

Descriptive data (with 95\% confidence intervals) presenting the types and quantities of each type of food and drink are provided overall and stratified by ethnicity. General linear regression models (PROC GLM) were then used to compare mean food and drink availability levels between homes with White British and Pakistani groups only (owing to insufficient numbers in the Other ethnicity category). The LSMEANS option was used to estimate the adjusted mean availability for both ethnic groups. Regression model 1 was unadjusted. Model 2 was adjusted for the total number of people reported to live in each household (un-weighted) as this has been shown to impact on home food availability previously ${ }^{(10,18)}$ and differs between the White and Pakistani families in this cohort $^{(16)}$. Full covariate adjustment was not deemed necessary here however, given the exploratory nature of the research. Data were analysed using the statistical software package SAS version $9 \cdot 2$.

\section{Results}

\section{Sample}

Of the 100 participants who agreed to take part, full food availability data were available from ninety-seven homes (whole categories of foods/drinks were missing from three participants' homes). There were similar numbers of White British ( $n$ 46, $47 \%)$ and Pakistani ( $n 41,42 \%)$ mothers, with fewer mothers from a combined ethnicity category defined as 'Other' ( $n$ 10, $11 \%$ ). Data from all three ethnic categories are provided for the descriptive, exploratory findings; however, only data from White British and Pakistani mothers were included in analyses comparing ethnic differences in food availability. Fifty-one per cent of mothers were normal weight at the booking appointment (approximately 12 weeks' gestation/pregnant), 29\% were overweight $\left(\mathrm{BMI} \geq 25 \mathrm{~kg} / \mathrm{m}^{2}\right)$ and $17 \%$ were obese $\left(\mathrm{BMI} \geq 30 \mathrm{~kg} / \mathrm{m}^{2}\right.$ ). Supplemental Table 1 (online supplementary material) shows the participants' characteristics.

\section{Presence/absence of foods/drinks in the bome}

Table 1 shows the frequency of homes that had at least one item of food or drink available within higher food categories. All homes had at least one type of fruit, at least one type of vegetable and at least one type of snack available. The majority of homes had at least one type of fresh fruit available and this was similar in all ethnicities. Availability of other forms of fruit (i.e. canned, dried and frozen) was less popular; however, around half of all homes had at least one type of canned or dried fruit. Availability of crisps/tortillas was also popular, with $80 \%$ and $90 \%$ availability in Pakistani and White British homes, respectively. Over $80 \%$ of White British and Pakistani homes also had at least one type of sweet biscuit available to them. Approximately $65 \%$ of White homes had at least one type of chocolate available, whereas less than $30 \%$ of Pakistani homes had chocolate available. Similarly, there were higher percentages of cakes and sweets in White homes. Approximately half of all homes had at least one type of ice cream available and this was similar across 
Table 1 Home food availability: frequency (\%) of the presence of foods within higher and sub-categories by ethnicity in a sample of families with infants approximately 18 months old from the Born in Bradford birth cohort, UK

\begin{tabular}{|c|c|c|c|c|c|c|c|c|}
\hline \multirow[b]{2}{*}{ Foods in the home } & \multicolumn{2}{|c|}{$\begin{array}{l}\text { White British } \\
\qquad(n \text { 46) }\end{array}$} & \multicolumn{2}{|c|}{$\begin{array}{l}\text { Pakistani } \\
(n 41)\end{array}$} & \multicolumn{2}{|c|}{$\begin{array}{l}\text { Other } \\
(n 10)\end{array}$} & \multicolumn{2}{|c|}{$\begin{array}{c}\text { All } \\
(n 97)^{\star}\end{array}$} \\
\hline & $n$ & $\%$ & $n$ & $\%$ & $n$ & $\%$ & $n$ & $\%$ \\
\hline \multicolumn{9}{|l|}{ Fruit } \\
\hline Fresh & 41 & $89 \cdot 1$ & 40 & $97 \cdot 6$ & 10 & $100 \cdot 0$ & 91 & $93 \cdot 8$ \\
\hline Canned/jar/tinned & 24 & $52 \cdot 2$ & 19 & $46 \cdot 3$ & 5 & $50 \cdot 0$ & 48 & $49 \cdot 5$ \\
\hline Dried & 28 & $60 \cdot 9$ & 22 & $53 \cdot 7$ & 5 & $50 \cdot 0$ & 55 & $56 \cdot 7$ \\
\hline Frozen & 3 & $6 \cdot 5$ & 0 & 0.0 & 1 & $11 \cdot 1$ & 4 & $4 \cdot 1$ \\
\hline Total fruit & 46 & $100 \cdot 0$ & 41 & $100 \cdot 0$ & 10 & $100 \cdot 0$ & 97 & $100 \cdot 0$ \\
\hline \multicolumn{9}{|l|}{ Vegetables } \\
\hline Fresh & 44 & $95 \cdot 7$ & 41 & $100 \cdot 0$ & 9 & $90 \cdot 0$ & 94 & $96 \cdot 9$ \\
\hline Canned/jar/tinned & 41 & $89 \cdot 1$ & 30 & $73 \cdot 2$ & 9 & $90 \cdot 0$ & 80 & $82 \cdot 5$ \\
\hline Frozen & 39 & $84 \cdot 8$ & 33 & $80 \cdot 5$ & 9 & $90 \cdot 0$ & 81 & $83 \cdot 5$ \\
\hline Total vegetables & 46 & $100 \cdot 0$ & 41 & $100 \cdot 0$ & 10 & $100 \cdot 0$ & 97 & $100 \cdot 0$ \\
\hline \multicolumn{9}{|l|}{ Snacks } \\
\hline Crisps/tortillas & 36 & $78 \cdot 3$ & 37 & $90 \cdot 2$ & 7 & $70 \cdot 0$ & 80 & $82 \cdot 5$ \\
\hline Sweet biscuits & 38 & $82 \cdot 6$ & 35 & $85 \cdot 4$ & 9 & $90 \cdot 0$ & 82 & $84 \cdot 5$ \\
\hline Salted nuts & 2 & $4 \cdot 4$ & 2 & 4.9 & 1 & $10 \cdot 0$ & 5 & $5 \cdot 2$ \\
\hline Chocolate & 30 & $65 \cdot 2$ & 11 & $26 \cdot 8$ & 4 & $40 \cdot 0$ & 45 & $46 \cdot 4$ \\
\hline Sweets & 22 & $47 \cdot 8$ & 17 & $41 \cdot 5$ & 5 & $50 \cdot 0$ & 44 & $45 \cdot 4$ \\
\hline Cakes & 22 & $47 \cdot 8$ & 14 & $34 \cdot 2$ & 3 & $30 \cdot 0$ & 39 & $40 \cdot 2$ \\
\hline Ice cream & 23 & $50 \cdot 0$ & 22 & $53 \cdot 7$ & 6 & $60 \cdot 0$ & 51 & $52 \cdot 6$ \\
\hline Total snacks & 46 & $100 \cdot 0$ & 41 & $100 \cdot 0$ & 10 & $100 \cdot 0$ & 97 & $100 \cdot 0$ \\
\hline \multicolumn{9}{|l|}{ Beverages } \\
\hline Sweetened drinkst & 28 & $60 \cdot 9$ & 35 & $85 \cdot 4$ & 5 & $50 \cdot 0$ & 68 & $70 \cdot 1$ \\
\hline Unsweetened drinksł & 14 & $30 \cdot 4$ & 10 & $24 \cdot 4$ & 2 & $20 \cdot 0$ & 26 & $26 \cdot 8$ \\
\hline Total drinks & 34 & $89 \cdot 5$ & 35 & $85 \cdot 4$ & 6 & $60 \cdot 0$ & 79 & $81 \cdot 4$ \\
\hline
\end{tabular}

*Includes Pakistani, White British and Other ethnicities.

tIncludes fizzy, sports and sweetened fruit drinks.

¥Unsweetened drinks are diet versions of sweetened drinks.

ethnicities (albeit somewhat lower in homes of 'Other' ethnicities). Approximately $70 \%$ of homes had sweetened beverages available, and non-sweetened beverages were found in $27 \%$ of all homes.

\section{Availability of individual foods}

Figures $1-4$ show the average availability of individual foods within the categories of fruit and vegetables by ethnicity. Fresh fruit commonly available in family homes were oranges, bananas, apples, satsumas and grapes. Commonly available fresh vegetables included potatoes, cucumber, tomatoes and carrots. The single greatest non-fresh fruit available in homes was raisins. Within the category of non-fresh vegetables, foods contributing the most were frozen mixed vegetables, tinned tomatoes and tinned peas. Crisps were the most commonly available type of snack food across all ethnic groups (data not shown).

\section{Ethnic comparisons}

Table 2 compares the availability of foods and drinks in homes of White British and Pakistani families. These analyses indicate that Pakistani homes had a greater availability of fresh fruits and sweetened drinks compared with White British homes, with more than twice the amount of these items available, even after adjustment for household size. Eighty-five per cent of Pakistani homes had sweetened beverages available, compared with $60 \%$ of White homes. Conversely, the proportion of homes with unsweetened (or 'diet') drinks available to them was lowest in the Pakistani homes (25\%) compared with White (31\%) and 'Other' ethnicity (78\%) homes. Availability of sugar-sweetened beverages in Pakistani homes was equivalent to approximately sixteen cans of fizzy drink per household on average, compared with an average of six in White British homes.

\section{Discussion}

Findings from the present exploratory study showed that all homes had some form of fruit and some form of vegetable available in them. More homes had fresh fruits and vegetables available compared with canned, frozen and dried fruits and vegetables. At least one type of snack food was also available in all of the homes in which inventories were conducted. Of these, crisps and biscuits were most likely to be available. Further exploration of the availability of individual foods showed that apples were available in the greatest quantity, with an average of between three and eight apples available in each home. The vegetable that was available in the greatest quantity was potatoes, with an average of approximately eight to twelve servings available in each home. Within non-fresh items, items that were available in the greatest quantities included raisins, 


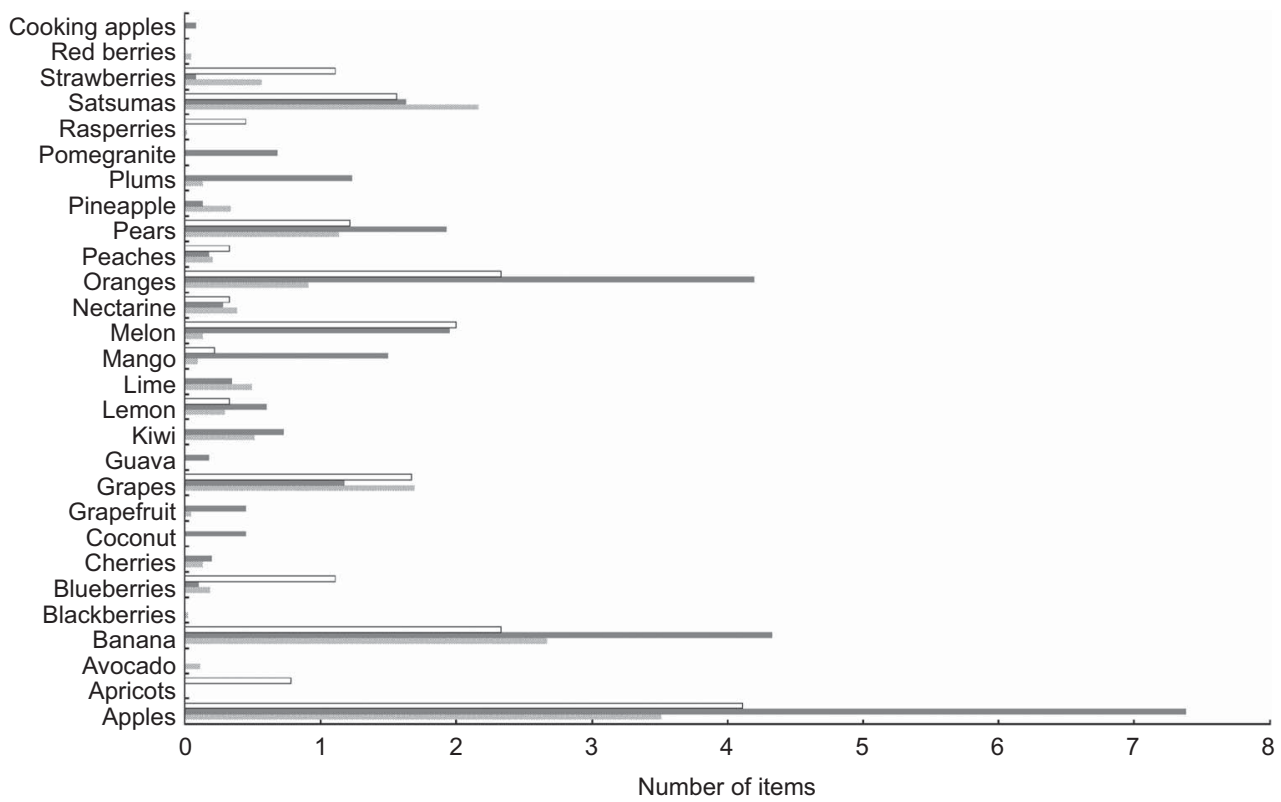

Fig. 1 Home availability of individual fresh fruits by ethnicity ( $\sqsubset$, Other; $\square$, Pakistani; $\cdots$, White British) in a sample of families with infants approximately 18 months old from the Born in Bradford birth cohort, UK

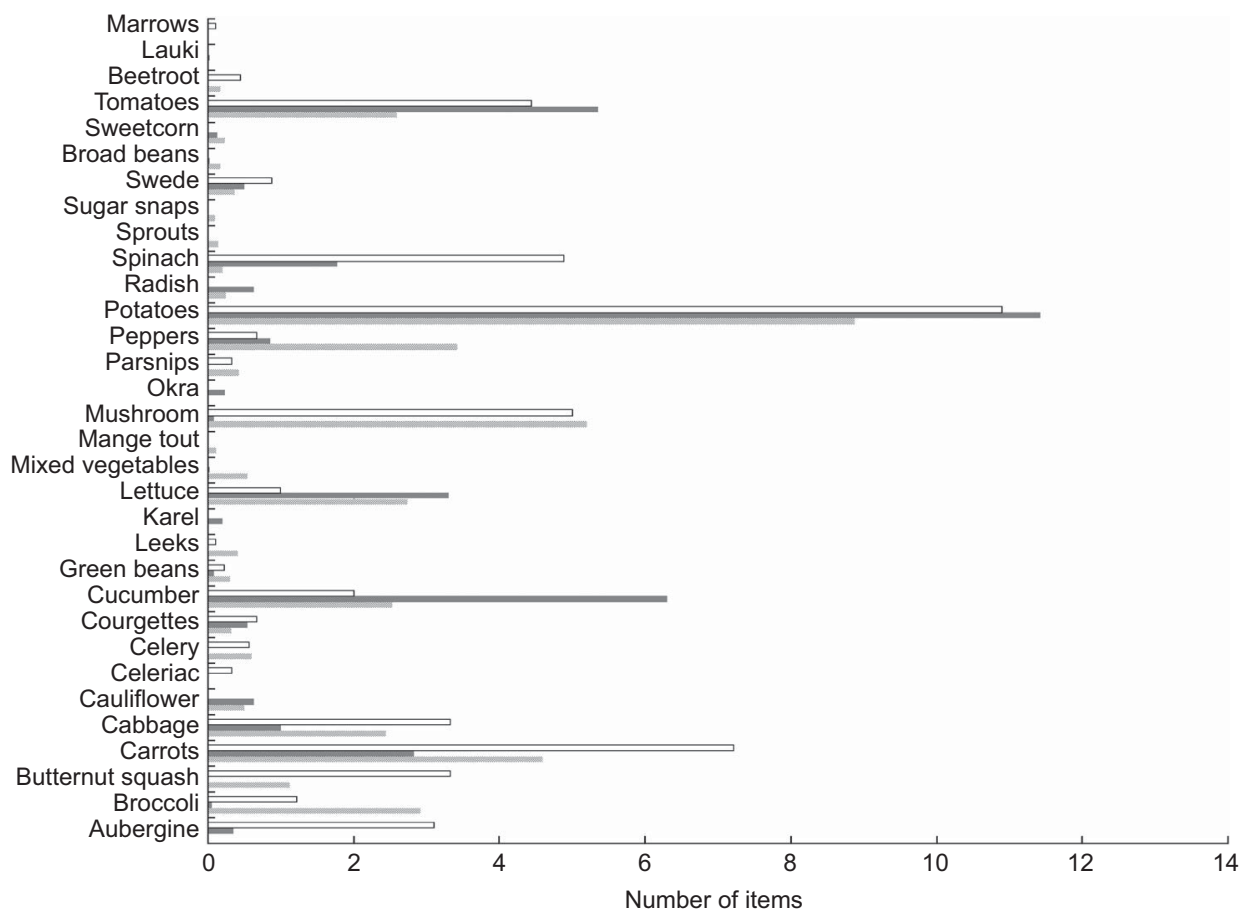

Fig 2 Home availability of individual fresh vegetables by ethnicity ( $\square$, Other; $\square$, Pakistani; $\approx$, White British) in a sample of families with infants approximately 18 months old from the Born in Bradford birth cohort, UK

frozen mixed vegetables and tinned tomatoes. Ethnic differences between homes of White British and Pakistani participants were found for the availability of fresh fruits and sugar-sweetened beverages, which were both found in higher amounts in Pakistani homes, even after adjustment for household size.
It is difficult to compare these findings with existing data, since there are currently no other comparable contemporary data on foods that are actually available within families' homes in the UK. The UK Office of National Statistics collects self-reported availability by asking families to report availability via purchasing habits. 


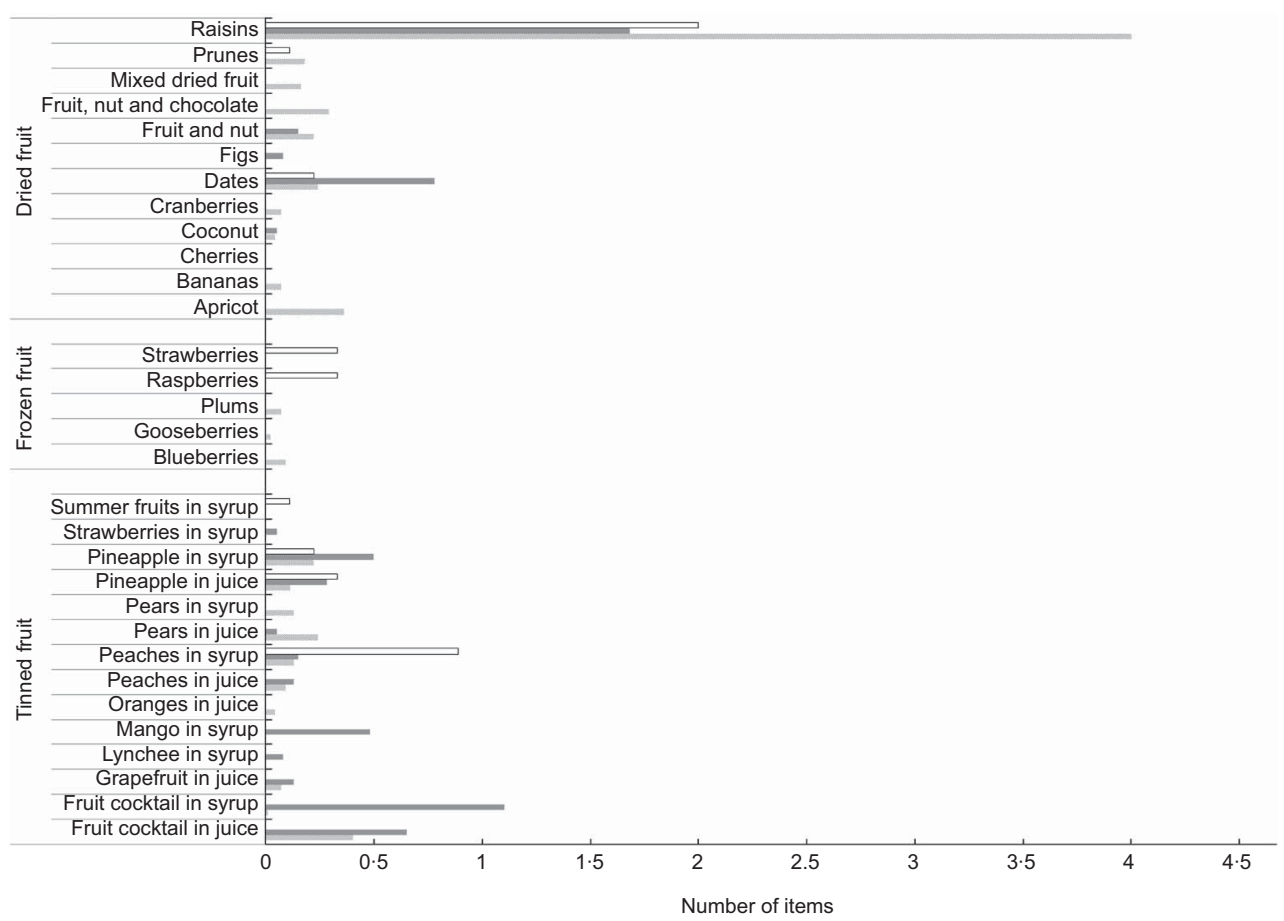

Fig. 3 Home availability of individual non-fresh fruits by ethnicity ( $\square$, Other; $\square$, Pakistani; with infants approximately 18 months old from the Born in Bradford birth cohort, UK

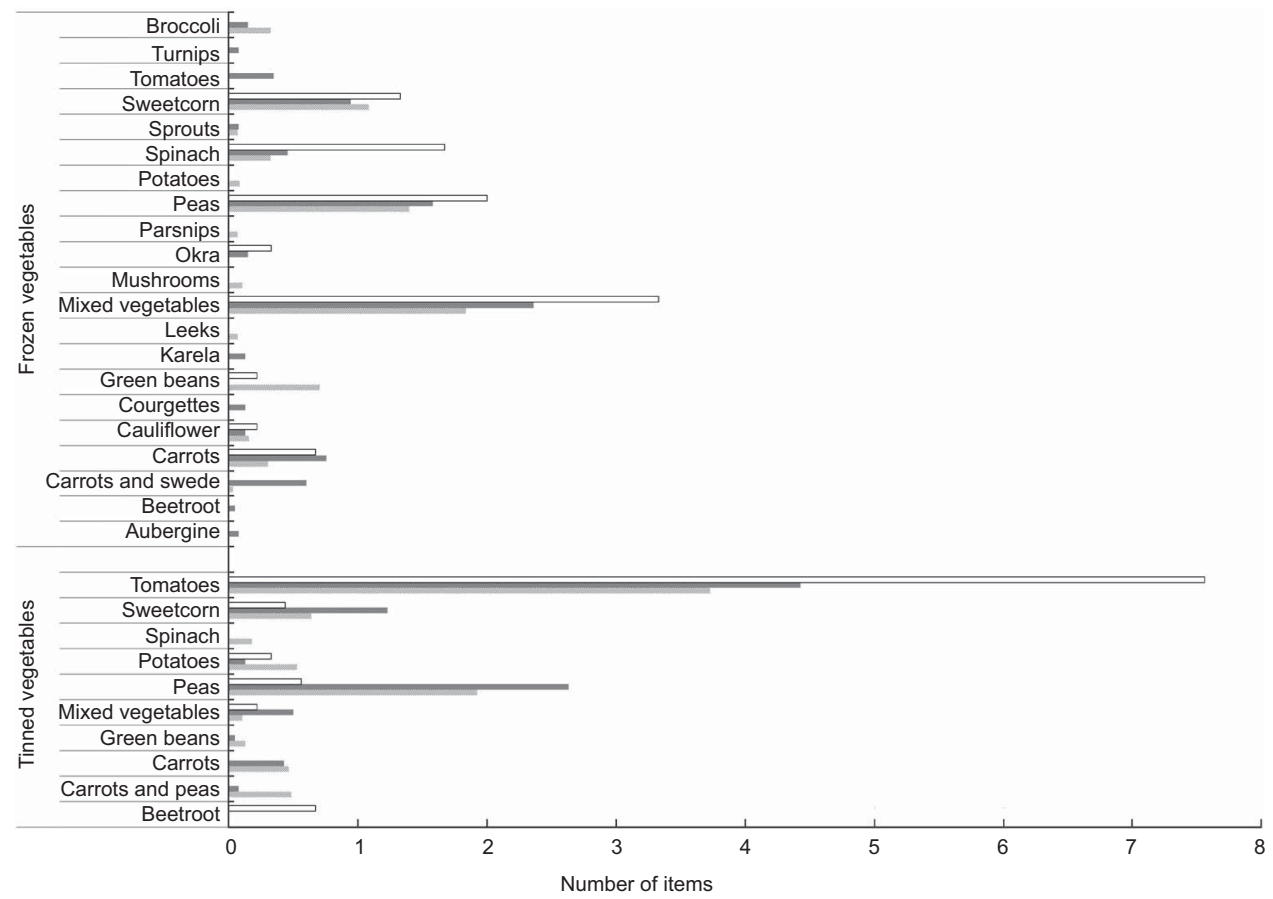

Fig. 4 Home availability of individual non-fresh vegetables by ethnicity ( $\square$, Other;, , Pakistani; $\cdots$, White British) in a sample of families with infants approximately 18 months old from the Born in Bradford birth cohort, UK

These data have been compared with data from other countries and indicate that UK households tend to have higher availability of cereals, but lower availability of fresh fruits and vegetables, than most of the ten other countries.
However, comparisons do not include availability of beverages and they do not indicate differences by participant characteristics ${ }^{(23)}$. A recent study in the USA indicates some differences in home food availability by ethnicity in which 
Table 2 Comparison of home food availability of White British and Pakistani mothers in a sample of families with infants approximately 18 months old from the Born in Bradford birth cohort, UK

\begin{tabular}{|c|c|c|c|c|c|c|c|c|}
\hline & \multicolumn{2}{|c|}{ White British ( $n$ 46) } & \multicolumn{2}{|c|}{ Pakistani ( $n$ 41) } & \multicolumn{2}{|c|}{ White British ( $n$ 46) } & \multicolumn{2}{|c|}{ Pakistani ( $n$ 41) } \\
\hline & \multicolumn{4}{|c|}{ Unadjusted } & \multicolumn{4}{|c|}{ Adjusted ${ }^{*}$} \\
\hline & Estimate & $95 \% \mathrm{Cl}$ & Estimate & $95 \% \mathrm{Cl}$ & Estimate & $95 \% \mathrm{Cl}$ & Estimate & $95 \% \mathrm{Cl}$ \\
\hline Fresh fruit (servings) & $15 \cdot 42 \dagger$ & $10 \cdot 79,20 \cdot 04$ & $29 \cdot 20$ & $24 \cdot 30,34 \cdot 09$ & $16 \cdot 04 t$ & $10 \cdot 98,21 \cdot 09$ & $28 \cdot 44$ & $23.95,33.94$ \\
\hline Fresh vegetables (servings) & $40 \cdot 39$ & $32 \cdot 17,48 \cdot 61$ & $36 \cdot 73$ & $28 \cdot 03,45 \cdot 44$ & $40 \cdot 47$ & $31 \cdot 47,49 \cdot 47$ & $36 \cdot 63$ & $26 \cdot 86,46 \cdot 41$ \\
\hline Tinned fruit & 1.59 & $0 \cdot 08,3 \cdot 10$ & $3 \cdot 49$ & $1 \cdot 89,5 \cdot 09$ & $1 \cdot 64$ & $-0.01,3.30$ & $3 \cdot 42$ & $1 \cdot 62,5 \cdot 21$ \\
\hline Tinned vegetables & $9 \cdot 56$ & $6 \cdot 25,12 \cdot 89$ & $10 \cdot 93$ & $7 \cdot 42,14 \cdot 43$ & $10 \cdot 07$ & $6 \cdot 45,13 \cdot 68$ & $10 \cdot 32$ & $6 \cdot 39,14 \cdot 25$ \\
\hline Frozen fruit & 0.22 & $-0.07,0.50$ & 0.00 & $-0.30,0.30$ & $0 \cdot 21$ & $-0 \cdot 10,0.52$ & 0.01 & $-0.32,0.35$ \\
\hline Frozen vegetables & $6 \cdot 72$ & $4 \cdot 72,8 \cdot 71$ & $7 \cdot 34$ & $5 \cdot 23,9 \cdot 45$ & $7 \cdot 02$ & $4 \cdot 84,9 \cdot 20$ & $6 \cdot 98$ & $4 \cdot 61,9 \cdot 34$ \\
\hline Cakes & $4 \cdot 46$ & $1.90,7 \cdot 02$ & 4.56 & $1 \cdot 85,7 \cdot 27$ & 4.99 & $2 \cdot 20,7 \cdot 78$ & 3.91 & $0.88,6.94$ \\
\hline Chocolate & $7 \cdot 33$ & $4.59,10.06$ & $3 \cdot 17$ & $0.28,6 \cdot 07$ & $6 \cdot 90$ & $3.91,9.88$ & $3 \cdot 69$ & $0.45,6.93$ \\
\hline Biscuits & $13 \cdot 57$ & $8 \cdot 70,18 \cdot 43$ & $15 \cdot 08$ & $9 \cdot 90,20 \cdot 20$ & $14 \cdot 95$ & $9 \cdot 67,20 \cdot 23$ & $13 \cdot 36$ & $7 \cdot 63,19 \cdot 10$ \\
\hline Ice cream & $3 \cdot 50$ & $1 \cdot 32,5 \cdot 68$ & $4 \cdot 90$ & $2 \cdot 59,7 \cdot 21$ & $4 \cdot 41$ & $2 \cdot 07,6 \cdot 75$ & $3 \cdot 80$ & $1 \cdot 26,6 \cdot 34$ \\
\hline Sweetened drinks & $5 \cdot 63+$ & $1 \cdot 57,9 \cdot 67$ & $16 \cdot 73$ & $12 \cdot 42,21 \cdot 04$ & $6 \cdot 89+$ & $2 \cdot 48,11 \cdot 29$ & $15 \cdot 20$ & $10 \cdot 42,19 \cdot 98$ \\
\hline Diet drinks & $2 \cdot 35$ & $0 \cdot 74,4 \cdot 00$ & $2 \cdot 41$ & $0 \cdot 71,4 \cdot 12$ & $2 \cdot 64$ & $0 \cdot 88,4 \cdot 40$ & $2 \cdot 06$ & $0.15,3.97$ \\
\hline
\end{tabular}

${ }^{*}$ Adjusted for total number of members in the household.

$+P<0.001$ comparing the availability between White British and Pakistani homes.

similar differences to those found here are reported, with a greater availability of fresh vegetables and soft drinks in the homes of Hispanic participants compared with African American homes ${ }^{(24)}$. However, these data were collected by self-report. Variability in the methods employed in these studies may well account for inconsistencies in findings. Studies measured using open, researcher-conducted inventories that have been published the last decade (i.e. since the last systematic review of home food availability measures $^{(8)}$ ) indicate some differences by weight status ${ }^{(22)}$ and provide evidence of a relationship between availability and dietary intake ${ }^{(25)}$. These provide support for the use of such methods in leading towards interventions to encourage optimising the healthfulness of foods and drinks available; however, they do not explore whether findings were dependent on ethnicity and both were conducted in the USA.

The present study indicates that availability of sugarsweetened beverages (predominantly fizzy drinks) was high in family homes, especially in Pakistani homes, with the equivalent of an average of sixteen cans per household and $85 \%$ having at least one sweetened drink available. Although evidence is not always clear, there is general support that consumption of sugar-sweetened beverages contributes significantly to obesity ${ }^{(19,26,27)}$. Data from randomised controlled trials support this work, with interventions targeting a reduction in sugar-sweetened beverages showing significant reductions in BMI compared with control groups ${ }^{(28,29)}$. Further, previous work indicates that this may also be linked to a greater odds of families consuming fast foods as part of their weekly family meals ${ }^{(30)}$. Some minority ethnic groups in the UK, including those of Pakistani origin, are more likely to experience poorer health outcomes, such as CVD and type 2 diabetes, compared with the White British population. The aetiology of this is likely to be multifaceted, including the impact of acculturation, genetic predisposition and access/use of health care, which are likely to impact on diet and other health behaviours. Data from a UK sample of mixed ethnicity showed that Pakistani boys (11-13 years) in particular were more likely to consume 'fizzy' drinks daily compared with White British boys. These data also suggest that Pakistani boys are less likely to meet targets for consuming 5-a-day for fruits and vegetables ${ }^{(31)}$. These availability data show that homes with participants of Pakistani origin had a higher availability of fresh fruits compared with White British homes. However, they do not provide details of the patterns of consumption by individual family members. Alarmingly, other data (not shown) from the BiB1000 study show a higher consumption of sugar-sweetened beverages in 18month-old infants born to Pakistani mothers compared with those born to White British parents after adjusting for mother's age and parental education $(\mathrm{OR}=2 \cdot 03$; $95 \% \mathrm{CI}$ $1 \cdot 53,2 \cdot 70)$. Consumption of water, however, was similar between infants of different ethnicity (OR $=1 \cdot 09$; $95 \% \mathrm{CI}$ $0 \cdot 84,1 \cdot 42)$.

Seasonality, in terms of the month in which inventories were completed, may have an impact on the foods available. Inventories were conducted every month over the period of 1 year, but there were fewer conducted during August and December due to staffing issues (coinciding with Ramadan and Christmas holidays). The influence of seasonality was considered by re-running analysis with adjustment for the month of data collection and did not change the findings; Pakistani homes had more fresh fruit and sugar-sweetened beverages than White British homes and no other foods were found to differ significantly between ethnicities (data not shown).

The impact of other variables such as socio-economic status on home food availability and their influence on the relationship between food availability and outcomes such as diet and obesity were not the focus of the current study. However, comparisons of food availability by maternal weight status did not identify any clear 
relationships (data not shown). Correlations with child BMI were not assessed in the 18-month-old infants but it is possible that the influence of home food availability on diet and BMI is greater in young children compared with adults, who are more likely to eat away from home ${ }^{(25)}$. Studies examining the relationship between home food availability and diet or BMI in children report inconsistent findings with variability in the strength of this relationship $^{(5-7,30,32)}$, although there is general agreement that the relationship is positive (especially for intake of vegetables). Few studies report the impact of socio-economic status. Ding et l $^{(7)}$ found an influence of household income, with more 'healthy' foods reported in homes with higher incomes. However, their study did not observe a reverse relationship with unhealthy foods. Clearly, more work is required to un-piece the explanatory factors and mediators that impact on the relationship between foods in the home and diet and health outcomes such as obesity.

Although the present exploratory study has a relatively small sample size, it is comparable (if not greater) to other studies that have collected home food availability data using direct observations by researchers. Previous work indicates that sixty-three households would be required per comparison group to detect a moderate difference of $50 \%$ of $1 \mathrm{SD}$ and only twenty-eight households per group would be needed to detect a difference as large as $75 \%$ of $1 \mathrm{SD}$ (based on one measurement per household) ${ }^{(18)}$. It is possible that other ethnic differences in availability might have been identified with a greater sample size. However, as there are currently no other studies that have measured, in depth, the types of foods and drinks within White British and Pakistani homes in the UK, the aim of the study was more exploratory in order to inform potential targets for the development of future interventions. It could also be argued that, due to the transitory nature of foods in the home (i.e. changing via purchasing and consumption), more than one visit would be required for accurate estimates of availability. Previous evidence actually suggests that the within-household variability of food availability is considerably lower than the between-household variability and that addition of multiple visits does not appreciably impact on estimates ${ }^{(18)}$. A further argument may be that participants changed their shopping habits in advance of the inventories due to social desirability. Following extensive data collection in a different cohort, this was found to be unlikely ${ }^{(10)}$ and when the participants were fully aware of the procedures (i.e. after they had already had completed an inventory), no efforts were made to change the environment for subsequent inventories, as within-house variability was very low ${ }^{(18)}$.

Knowledge of the types and quantities of foods and drinks available in family homes supports the development of targeted intervention programmes wishing to improve the foods available within family homes for obesity prevention or management, or for overall diet improvement. This has the ability to identify population subgroups at nutritional risk and implement appropriate health promotion and disease prevention programmes. Descriptive data shown here indicate that potential targets might be: (i) promoting the variety, availability and quantity of all types of fruits and vegetables (e.g. encouraging purchase of tinned/frozen fruit in addition to fresh fruit); (ii) reducing purchase of crisps and biscuits (which were both available in over $80 \%$ of homes); and (iii) discouraging purchase of sweetened beverages, especially within homes of Pakistani mothers (in which $85 \%$ of homes had at least one type of sweetened beverage available). This information has been fed into a needs assessment stage of an intervention mapping process in which a culturally appropriate obesity prevention intervention has been developed in Bradford (a city in the north of the UK).

\section{Acknowledgements}

Sources of funding: The work was funded by an NIHR CLAHRC implementation grant and an NIHR applied programme grant (RP-PG-0407-10044). This paper presents independent research commissioned by the National Institute for Health Research (NIHR) under the CLAHRC programme. The views expressed are those of the authors and not necessarily those of the National Health Service, the NIHR or the Department of Health. Conflict of interest: The authors declare that they have no conflicts of interest. Authors' contributions: M.B. designed the study, coordinated data collection, and led analysis and writing of the article. P.S. and A.H. provided expertise in designing the study and contributed to writing the article. G.S. supervised the analysis and contributed to writing the article. Acknowledgments: The authors are grateful to all the families who took part in the study, to the midwives for their help in recruiting them, the paediatricians and health visitors, and to the BiB team which included interviewers, data managers, laboratory staff, clerical workers, research scientists, volunteers and managers.

\section{Supplementary material}

To view supplementary material for this article, please visit http://dx.doi.org/10.1017/S1368980014000147

\section{References}

1. Pearson N, Biddle SJ \& Gorely T (2008) Family correlates of fruit and vegetable consumption in children and adolescents: a systematic review. Public Health Nutr 12, 267-283.

2. Rosenkranz RR \& Dzewaltowski DA (2008) Model of the home food environment pertaining to childhood obesity. Nutr Rev 66, 123-140.

3. van der Horst K, Oenema A, Ferreira I et al. (2007) A systematic review of environmental correlates of obesityrelated dietary behaviors in youth. Health Educ Res 22, 203-226.

4. Rasmussen M, Krolner R, Klepp K-I et al. (2006) Determinants of fruit and vegetable consumption among 
children and adolescents: a review of the literature. Part I: quantitative studies. Int J Behav Nutr Phys Act 3, 22.

5. Arcan C, Hannan PJ, Fulkerson JA et al. (2013) Associations of home food availability, dietary intake, screen time and physical activity with BMI in young American-Indian children. Public Health Nutr 16, 146-155.

6. Boles RE, Scharf C, Filigno SS et al. (2013) Differences in home food and activity environments between obese and healthy weight families of preschool children. $J$ Nutr Educ Behav 45, 222-231.

7. Ding D, Sallis JF, Norman GJ et al. (2012) Community food environment, home food environment, and fruit and vegetable intake of children and adolescents. J Nutr Educ Behav 44, 634-638.

8. Bryant M \& Stevens J (2006) Measurement of food availability in the home. Nutr Rev 64, 67-76.

9. Bryant M, Ward D, Hales D et al. (2008) Reliability and validity of the Healthy Home Survey: a tool to measure factors within homes hypothesized to relate to overweight in children. Int J Behav Nutr Phys Act 5, 23.

10. Stevens J, Bryant M, Wang L et al. (2011) Exhaustive measurement of food items in the home using a universal product code scanner. Public Health Nutr 14, 314-318.

11. Sisk C, Sharkey J, McIntosh W et al. (2010) Using multiple household food inventories to measure food availability in the home over 30 days: a pilot study. Nutr J9, 19.

12. Krukowski R, Harvey-Berino J \& West D (2010) Differences in home food availability of high- and low-fat foods after a behavioral weight control program are regional not racial. Int J Behav Nutr Phys Act 7, 69.

13. Campbell KJ (2007) Associations between the home food environment and obesity-promoting eating behaviors in adolescence. Obesity (Silver Spring) 15, 719-730.

14. Hanson N, Neumark-Sztainer D, Eisenberg M et al. (2005) Associations between parental report of the home food environment and adolescent intakes of fruit, vegetables and dairy foods. Public Health Nutr 8, 77-85.

15. Spurrier NJ, Magarey AA, Golley RK et al. (2008) Relationships between the home environment and physical activity and dietary patterns of preschool children: a cross sectional study. Int J Behav Nutr Phys Act 5, 31.

16. Bryant M, Santorelli G, Fairley L et al. (2013) The Born in Bradford Childhood Obesity Scientific Group. Design and characteristics of a new birth cohort to study the early origins of childhood obesity: the BiB1000 study. Longit Life Course Stud 4, 119-135.

17. Wright J, Small N, Raynor P et al. (2013) Cohort profile: the Born in Bradford multi-ethnic family cohort study. Int $J$ Epidemiol 42, 978-991.

18. Stevens J, Bryant M, Wang C-H et al. (2012) Sample size and repeated measures required in studies of foods in the homes of African-American families. J Nutr $\mathbf{1 4 2}$, 1123-1127.

19. Malik VS, Schulze MB \& Hu FB (2006) Intake of sugarsweetened beverages and weight gain: a systematic review. Am J Clin Nutr 84, 274-288.

20. Nicklas TA, Yang S-J, Baranowski T et al. (2003) Eating patterns and obesity in children: the Bogalusa Heart Study. Am J Prev Med 25, 9-16.

21. Jago R, Page A, Froberg K et al. (2008) Screen-viewing and the home TV environment: the European Youth Heart Study. Prev Med 47, 525-529.

22. Byrd-Bredbenner C \& Maurer Abbot J (2009) Differences in food supplies of US households with and without overweight individuals. Appetite 52, 479-484.

23. Byrd-Bredbenner C, Lagiou P \& Trichopoulou A (2000) A comparison of household food availability in 11 countries. $J$ Hum Nutr Diet 13, 197-204.

24. Skala K, Chuang R-J, Evans A et al. (2012) Ethnic differences in the home food environment and parental food practices among families of low-income Hispanic and African-American preschoolers. J Immigr Minor Health 14, 1014-1022.

25. Bryant M, Stevens J, Wang L et al. (2011) Relationship between home fruit and vegetable availability and infant and maternal dietary intake in African-American families: evidence from the exhaustive home food inventory. $J \mathrm{Am}$ Diet Assoc 111, 1491-1497.

26. Pereira MA (2006) The possible role of sugar-sweetened beverages in obesity etiology: a review of the evidence. Int J Obes (Lond) 30, Suppl. 3, S28-S36.

27. Malik VS, Willett WC \& Hu FB (2009) Sugar-sweetened beverages and BMI in children and adolescents: reanalyses of a meta-analysis. Am J Clin Nutr 89, 438-439.

28. Sichieri R, Yokoo EM, Pereira RA et al. (2013) Water and sugar-sweetened beverage consumption and changes in BMI among Brazilian fourth graders after 1-year follow-up. Public Health Nutr 16, 73-77.

29. James J, Thomas P, Cavan D et al. (2004) Preventing childhood obesity by reducing consumption of carbonated drinks: cluster randomised controlled trial. BMJ 328, 1237.

30. Boutelle KN, Fulkerson JA, Neumark-Sztainer D et al. (2007) Fast food for family meals: relationships with parent and adolescent food intake, home food availability and weight status. Public Health Nutr 10, 16-23.

31. Harding S, Teyhan A, Maynard MJ et al. (2008) Ethnic differences in overweight and obesity in early adolescence in the MRC DASH study: the role of adolescent and parental lifestyle. Int J Epidemiol 37, 162-172.

32. Cullen K, Baranowski T, Klesges L et al. (2004) Anthropometric, parental, and psychosocial correlates of dietary intake of African-American girls. Obes Res 12, Suppl., 20S-31S. 